\title{
High-sensitivity EUV Resists based on Tetrafluoroethylene contained Fluoropolymers
}

\author{
Tsuneo Yamashita ${ }^{1}$, Masamichi Morita ${ }^{1}$, Yoshito Tanaka ${ }^{1}$, \\ Julius Joseph Santillan ${ }^{2}$, and Toshiro Itani ${ }^{2}$ \\ ${ }^{1}$ Chemical $R \& D$ Center, Daikin Industries, Ltd., \\ 1-1 Nishi-Hitotsuya, Settsu,Osaka, 566-8585 Japan \\ ${ }^{2}$ Semiconductor Leading Edge Technologies, Inc. \\ 16-1 Onogawa, Tsukuba, Ibaraki 305-8569, Japan
}

\begin{abstract}
There is a growing interest in the fluorinization of resist materials in improving pattern formation efficiency for extreme ultraviolet (EUV) lithography. The increased polymer absorption coefficient obtained through this resist platform is expected to enhance acid production and in effect improve pattern formation efficiency. Our work over the past several years has shown that the main-chain fluorinated base resins realized by the co-polymerization of tetrafluoroethylene (TFE) and norbornene derivatives offer high dissolution rates. Based on this, a EUV resist which was prepared using the fluorinated polymers was investigated. Imaging evaluations, using the small field exposure tool (SFET by Canon / EUVA) with annular ( $\sigma$ outer $0.7 / \sigma$ inner 0.3 ) illumination conditions were performed. Relatively high sensitivity of $6.3 \mathrm{~mJ}^{\circ} \mathrm{cm}^{-2}$ for half-pitch (hp) $45 \mathrm{~nm}$ and satisfactory resolution limit of hp $40 \mathrm{~nm}$ was achieved. At present, line width roughness (LWR) was measured at comparatively large values of more than $8.4 \mathrm{~nm}$ at hp $45 \mathrm{~nm}$. This shows that further material and process optimizations may be necessary to improve its present lithographic capability. However, these initial results have shown the potential of fluorinated-polymer based platform as a possible solution for high sensitivity, high resolution and low LWR EUV resists. In this paper, we report recent results of high sensitivity of $5.1 \mathrm{~mJ} \cdot \mathrm{cm}^{-2}$ for half-pitch (hp) $40 \mathrm{~nm}$, optimization of protecting groups and photo acid generators.
\end{abstract}

Keywords: EUV. extreme ultraviolet lithography, resist, fluoropolymers, Quartz Crystal Microbalance (QCM), fluoroalcohol

\section{Introduction}

Extreme ultraviolet lithography (EUVL) is considered as the most promising technology for mass production of semiconductor devices beyond the $22 \mathrm{~nm}$ node. This was made possible by the worldwide cooperation[1-5] of industry consortia, national laboratories, universities, suppliers and device manufacturers. However, many challenges remain for the industry to understand clearly and to overcome before EUVL will be ready for high-volume device manufacture. Efforts have been made to improve the various critical components of EUVL, such as light source,[6,7] exposure too,[8,9] mask,[10] resist material,[11-13] and so on. Among these components, the resist materials and processing are considered as one of the most critical issues. EUV resist development is governed by three factors; sensitivity, resolution limit and line-width roughness (LWR). Aside from the obvious resolution targets, EUV resist must show a high sensitivity, to compensate for the low power of EUV sources and improve throughput. It must also show a low value of LWR at these fine patterns.[14] However, these three have been known to have trade-off relationships and consequently, should be achieved concurrently.[12,13,15) Various methods in material development have been presented focusing on the use of molecular,[16,17] Photoacid generator (PAG) bound resists, $[18,19]$ and so on. Another possible solution being presented in 
simultaneously meeting these requirements is through the increase in pattern formation efficiency.[20] For the efficient use of the energy of incident radiation, the absorption coefficient of resist films must be increased. For deep-UV (DUV) resists, absorption coefficient has been optimized by adjusting the concentration or absorption coefficient of PAGs.[21,22] This is because the direct excitation of PAGs by incident photons is a major acid generation path in DUV resists. However, for EUV resists, acids generators are mainly sensitized by secondary electrons.[23] With the continuous development of EUV resists, sensitization mechanisms were identified and understood. It was thus noted, that the polymer absorption of EUV resists must be increased.[23) To increase polymer absorption, there is a growing interest in the introduction of fluorine atoms to resist materials for EUVL.[24] Recent study of fluorinated-polymers has shown that introduction of fluorine atoms can enhance acid generation yields under EUV exposure. This result was due to the increase in absorption coefficient by the introduction of fluorine atoms to the polymer chain.[20] Flourinated-polymer based resists, which have been extensively investigated in the past for use in 157-nm lithography,[25-27] easily provide the necessary platform to provide high absorption coefficient EUV resists. In this work, the application and evaluation of fluorinated-polymer based EUV resists in achieving; high sensitivity, good resolution limit and improved LWR are discussed. In 2009, we had first reported about main-chain fluorine containing polymers (we coaled TFE/NB1FVIP) that were able to use with EUV resist. Imaging evaluations, using the small field exposure tool (SFET by Canon / EUVA) with annular ( $\sigma$ outer $0.7 / \sigma$ inner 0.3 ) illumination conditions were performed. Relatively high sensitivity of $6.3 \mathrm{~mJ} \cdot \mathrm{cm}^{-2}$ for half-pitch (hp) $45 \mathrm{~nm}$. further material and process optimizations may be necessary to improve its present lithographic capability. In theis conference, we report the recent results of high sensitivity of $5.1 \mathrm{~mJ} \cdot \mathrm{cm}^{-2}$ for half-pitch (hp) $40 \mathrm{~nm}$, optimazation of protecting groups and photo acid generator.

\section{Experimental}

\subsection{Materials}

\subsubsection{Synthesis of monomer}

All materials were obtained from commercial suppliers and used without further purification, unless otherwise noted. Functional norbornene was prepared by the cyclo-addition of cyclopentadiene and a-Fluoro-acryl fluoride as described in the literature [33.35]

\subsubsection{Synthesis of monomer}

1,1,1,3,3,3-Hexafluoro-2-(2-fluoro-bicyclo[2.2.1]h ept-5-en-2-yl)-propan-2-ol (2)

2-Fluoro-bicyclo[2.2.1] hept-5-ene-2-carbonyl fluoride (1) was added to the suspension of dry KF and dry CsF in anhydrous THF under nitrogen. Trifluoromethyltrimethylsilane was slowly dropped into the mixture under nitrogen at $10{ }^{\circ} \mathrm{C}$. The reaction mixture was stirred at room temperature overnight. After reaction, $500 \mathrm{ml}$ of $1.0 \mathrm{~N} \mathrm{HCl} \mathrm{aq} \mathrm{was} \mathrm{added} \mathrm{to} \mathrm{the} \mathrm{mixture} \mathrm{and}$ extracted with $500 \mathrm{ml}$ diethyl ether. The combined ether extracts were washed with $1.0 \mathrm{~N} \mathrm{HCl}$ aq, water and brine. The ether solution was dried over $\mathrm{MgSO}_{4}$, evaporated and distilled at $60{ }^{\circ} \mathrm{C}$ at 5 $\mathrm{mmHg}$ to yield $200 \mathrm{~g}(90 \%)$ of functional norbornene as colorless oil (Schem 1).

\subsubsection{Radical polymerization}

The typical polymerization procedure is as follows. A 100-ml stainless-steel pressure vessel was charged with a solution of monomers, solvents, and initiator. The vessel was closed, purged with nitrogen, cooled, evacuated, and charged with TFE monomer. Radical copolymerization were carried out at $40{ }^{\circ} \mathrm{C}$ and maintained for 18-24 hrs. The polymer solution was poured into hexane, and was precipitated and washed by using a large excess of hexane. The resulting polymer powder was dried in vacuum.

\subsection{Characterization}

The polymer structures and compositions were determined from the NMR spectra. Namely, $1 \mathrm{H}$ and 19F NMR spectra were obtained at room temperature in acetone-d6 or $\mathrm{CDCl}_{3}$ on a Brucker AC-300P FT-NMR spectrometer. The polymer structures were also confirmed by FT-IR spectra, which were captured by a PERKIN ELEMER 1760X FT-IR spectrometer.

The weight-average molecular weight (MW) and number-average molecular weight $(\mathrm{Mn})$ were determined by gel permeation chromatography (GPC, GPC-104 SHOWA DENKO K. K.) using refractive-index (RI) data.

UV measurements at $193-\mathrm{nm}$ wavelength were performed using a VUV-VASE spectroscopic 

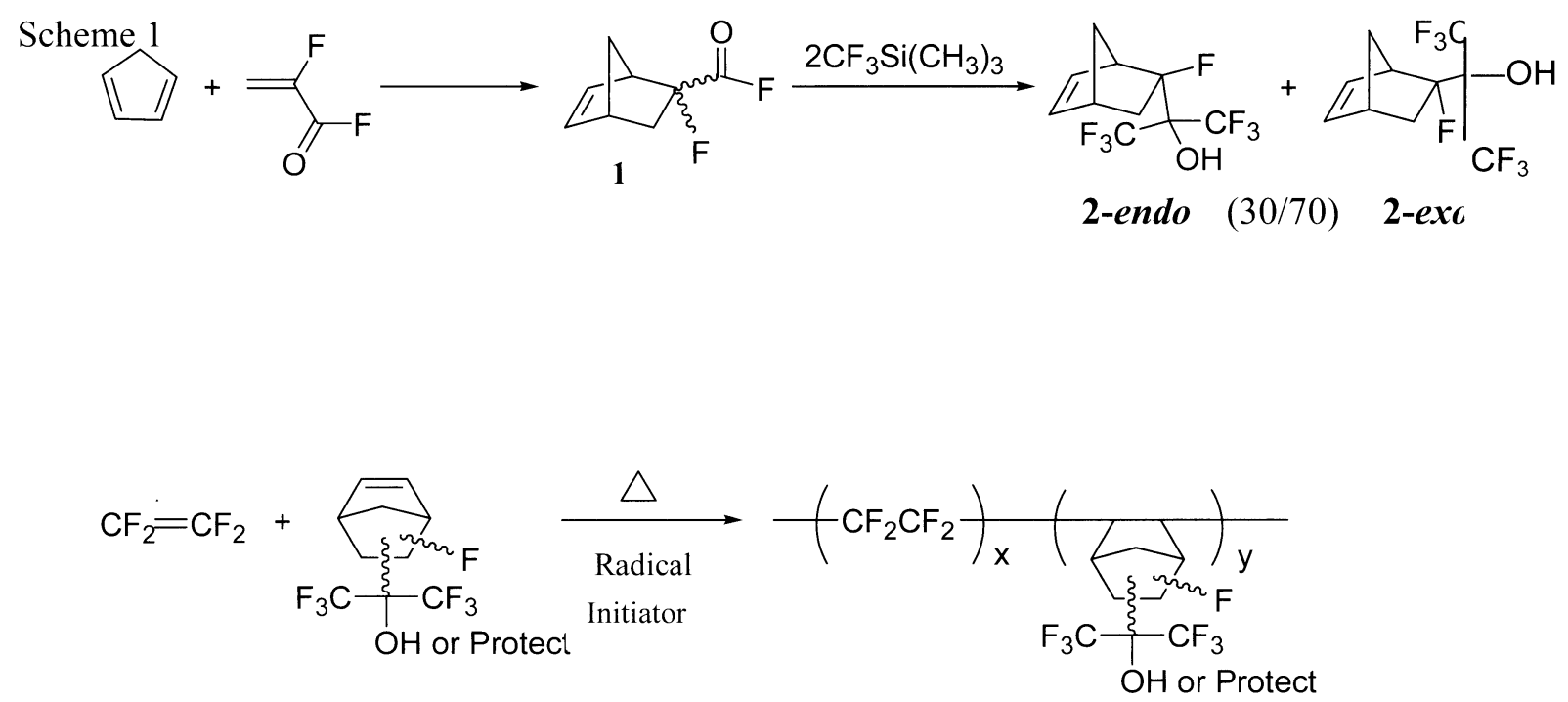

ellipsometer (M-2000D, J. A. Woollam Co., Inc.). Sample films were cast on a $\mathrm{CaF}_{2}$ plate from solutions and baked at $110{ }^{\circ} \mathrm{C}$ for 90 seconds prior to measurements. Film thickness was measured by using an interferometer. All absorbance data reported are in base 10 .

The dissolution behavior (i.e. the dissolution kinetics of the polymer film) during the development of the film was studied using a quartz crystal microbalance (QCM, Maxtek Model TM-400) in a fashion similar to a reported procedure [34]. The QCM instrumentation can, in combination with a compensated phase-locked oscillator circuit, measure the changes in film mass and motional resistance simultaneously during development. The developer used was a $0.262 \mathrm{~N}$ tetramethylammonium hydroxide (TMAH) aqueous solution (PD-523) supplied by Japan Synthetic Rubber Co., Ltd.

\subsection{Resist materials}

TFE/NB1FVIP was easily protected the functional group that polymer reaction by using an alkyl chloromethyl ether with presence of organic amine or alkali metal hydride as a base. Triphenylsulfonium perfluoro 1-butanesulfonate (TPS-PFBS) was utilized as PAG. Various quenchers such as tri-n-hexylamine (THA), triethanolamine (TEA) and trioctylamine (TOA) were used for lithographic performance optimization. For the solvent methyl amyl ketone (MAK) was utilized.[28]

The absorption coefficient of the fluorinated-polymer based resist at EUV was theoretically estimated from the sum of the atomic absorption cross sections of the atoms contained in the polymer and a set constant polymer-density (1.2 $\mathrm{g} \cdot \mathrm{cm}^{-3}$ for typical poly(methyl methacrylate) (PMMA) resist).[29] Improved absorption coefficient was calculated at $8.3 \mu^{-1}$ for the fluorinated-polymer based resist in comparison to $4.3 \mu \mathrm{m}^{-1}$ obtained from a typical poly(hydroxystyrene)(PHS) based resist. This shows fairly high absorption coefficient of the fluorinated-polymer EUV resist.

Lithographic exposure experiments were performed at a resist film thickness of $60 \mathrm{~nm}$ on a Si substrate processed with hexamethyldisilazane (HMDS) for improved adhesion. The post application bake (PAB) was $110{ }^{\circ} \mathrm{C}$ for $60 \mathrm{~s}$ while the post exposure bake (PEB) was $100^{\circ} \mathrm{C}$ for $90 \mathrm{~s}$. After exposure, development using $0.26 \mathrm{~N}$ tetramethylammonium hydroxide (TMAH) was done for $60 \mathrm{~s}$.

\subsection{Tool condition}

To minimize the possibility of contamination of the EUV exposure tool, the screening of resist outgassing is performed. In-house resist outgassing evaluations[30] were made at ultrahigh vacuum conditions 10-7 $\mathrm{Pa}$. An ion gauge is used for the pressure rise method while quadropole mass spectrometry (QMS) at a range of 1-200 amu was utilized for the accurate identification of resist outgassing elements released upon EUV exposure.

Figure 1 shows the small field exposure tool (SFET; EUVA/Canon) with 0.3 numerical aperture (NA) and annular ( $\sigma$ outer $0.7 / \sigma$ inner 0.3 ) 


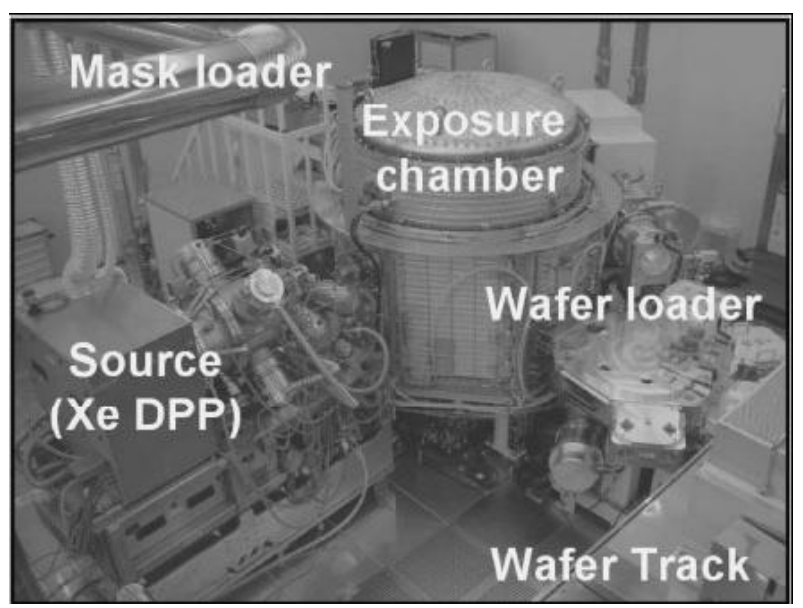

Fig. 1. The small field exposure tool or SFET with $0.3 \mathrm{NA}$ and $\operatorname{annular}(\sigma$ outer $0.7 / \sigma$ inner 0.3$)$ illumination conditions where all imaging exposureswere performed.

illumination conditions where all imagingexposures were performed. Frame exposures for resist sensitivity analysis were also done using the SFET with the same exposure conditions. The SFET is linked to the coater/developer track system ACT-12 (Tokyo Electron) in a chemically controlled environment. Line and space (L/S) pattern top-view pictures, feature size and LWR measurements were obtained using a Hitachi S9380II scanning electron microscope (SEM). LWR measurements were performed at a magnification of $200 \mathrm{~K}$, inspection area of $471 \mathrm{~nm}$, sum line/point at $22 \mathrm{~nm}$ and threshold at $50 \%$.

\section{Results and Discussion}

3.1. Synthesis of monomer and polymer, and measurement results of dissolution rate

The main-chain-fluorinated polymer is characterized by the functional norbornenes. Functional group incorporation relieves norbornenes from their large hydrophobicity. The alkaline-soluble ability depends on their acidities by introducing fluorine at the neighborhood of the HFA group. Their properties improve the adhesion to the substrate during the spin-coating process and wettability, solubility during the development process, etc..

Functional norbornene was prepared in 2 steps in $90 \%$ yield, as shown in Schem 1. The cyclo-addition (Diels-Alder reaction) of cyclopentadiene with 2-fluoro-acryloyl fluoride was carried out in bulk at the room temperature. The reaction proceeded smoothly. 2 was easily obtained by the reaction of 1 with trifluoromethyltrimethylsilane $\mathrm{CF}_{3} \mathrm{Si}\left(\mathrm{CH}_{3}\right)_{3}$ of 2 equivalents in THF at the room temperature. The ratio of endo/exo of isomer was 30/70 after the distillation. It is known that the Diels-Alder reaction of cyclopentadiene with $\alpha$-fluoro-acrylate derivatives usually gives exo-rich addition product, but the Diels-Alder reaction of acrylate derivatives provides endo-rich adduct (Schem 1).

The TFE/functional norbornene polymers are easily synthesized in the solution of TFE and functional norbornene with radical initiators. The resulting copolymer was found to have $\mathrm{Tg}$ of 130 ${ }^{\circ} \mathrm{C}$.

\subsection{Reactivity of protecting group}

The HFA group is easily protected by the conventional protective groups. Protection raises resist material sensitivity and imaging performance. Partial protection of the TFE/NB1FVIP with alkyl chloromethyl ethers was carried out by polymer reaction using an amine as a base.

\subsection{Resist outgassing evaluations}

After resist outgassing analysis of the TFE/NB1FVIP based EUV resist, comparatively minimal resist outgassing rate was measured $(1.3 \times$ 10-17 molecules $\left.\cdot \mathrm{cm}^{-2} \cdot \mathrm{s}_{-1}\right)$ and hydrofluoric acid, which is viewed as a possible harmful source of contamination[31] were also not detected with QMS analysis.

\subsection{Sensitivity evaluations}

Resist sensitivity analysis was performed with exposure doses ranging from 0.1 to $6 \mathrm{~mJ} / \mathrm{cm}^{2}$. Figure 2 shows the sensitivity curve of the fluorinated-polymer based resist in comparison to a high performance EUV resist, a copolymer of PHS with a high activation energy (Ea) blocking group, Selete standard resist 3 (SSR3). Relatively high sensitivity was obtained compared to high performance SSR3. The clearing dose $\left(E_{0}\right)$ of the fluorinated-polymer based resist was $2.4 \mathrm{~mJ} / \mathrm{cm}^{2}$ while the $\mathrm{E}_{0}$ of the $\mathrm{SSR} 3$ was $4.6 \mathrm{~mJ} / \mathrm{cm}^{2}$.

\subsection{Imaging and lithographic performance}

\subsubsection{Previous work}

Initial lithographic exposures using the SFET have shown the imaging capability of the fluorinated-polymer based EUV resist with THA as quencher. The lithographic performance of the fluorinated-polymer based EUV resist. At 


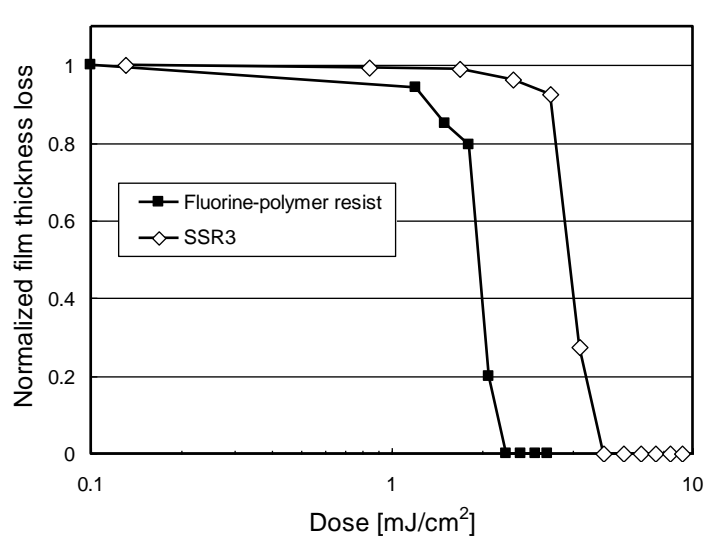

Fig. 2. The sensitivity curve of the fluorinated-polymer (TFE/NB1FVIP) based resist in comparison to the high performance Selete standard resist 3 (SSR3). to the occurrence of bending due to pattern instability or collapse. Quencher was changed to TEA as a possible solution in improving resolution and LWR.

As discussed in the previous chapter, one common issue reoccurring in this initial work on fluorinated-polymer based EUV resist is pattern instability at finer pattern sizes below hp $45 \mathrm{~nm}$. Relatively weak resist adhesion on the $\mathrm{Si}$ wafe surface is viewed as one possible cause. Some groups have reported the application of an underlayer in improving pattern stability.[32]

An organic spin-on underlayer was used at a $20 \mathrm{~nm}$ thickness to confirm the applicability of this concept on TFE/NB1FVIP based EUV resists with TEA as quencher. Figure 3 shows the lithographic performance of the TFE/NB1FVIP based EUV

\begin{tabular}{|c|c|c|c|c|c|c|}
\hline Pattern size & $50 \mathrm{~nm}$ & $45 \mathrm{~nm}$ & $40 \mathrm{~nm}$ & $35 \mathrm{~nm}$ & $32 \mathrm{~nm}$ & $30 \mathrm{~nm}$ \\
\hline $\begin{array}{c}\text { Top-view } \\
\text { SEM } \\
\text { imaqes }\end{array}$ & Dose: $6.7 \mathrm{~mJ} / \mathrm{cm}^{2}$ & $\begin{array}{l}7.1 \mathrm{~mJ} / \mathrm{cm}^{2} \\
\text { LWR: } 10 \mathrm{~nm}\end{array}$ & $7.5 \mathrm{~mJ} / \mathrm{cm}^{2}$ & $8.3 \mathrm{~m} / \mathrm{cm}^{2}$ & $8.3 \mathrm{~mJ} / \mathrm{cm}^{2}$ & $\begin{array}{c}8.3 \mathrm{~mJ} / \mathrm{cm} \\
\mathrm{cm}\end{array}$ \\
\hline
\end{tabular}

Fig. 3. The lithographic performance of the TFE/NB1FVIP based EUV resist with the application of underlayer.

half-pitch (hp) $45 \mathrm{~nm}$, a significantly high sensitivity of $6.3 \mathrm{~mJ} / \mathrm{cm} 2$ was obtained.

However, in the same pattern size, a large LWR of $12.9 \mathrm{~nm}$ was observed. Moreover, resolution was limited at roughly hp $40 \mathrm{~nm}$ with some obvious issues on pattern stability and residual resist or scumming between the lines. These initial results show that high sensitivity is possible with the TFE/NB1FVIP based EUV resist but optimization focusing on LWR and resolution capability is necessary. The protecting ratio of the fluorinated-polymer was varied for optimization. As a result, the lithographic performance of the fluorinated-polymer based EUV resist at optimized protecting ratio of the polymer. In comparison to the initial formulation, a slight decrease in resist sensitivity at hp $45 \mathrm{~nm}$ (dose: $\left.7.1 \mathrm{~mJ} / \mathrm{cm}^{2}\right)$ was observed. However, an improved LWR $(8.4 \mathrm{~nm}$ at hp $45 \mathrm{~nm}$ ) and decrease in residue or scumming was obtained. Resolution limit remains at $\mathrm{hp} 40$ $\mathrm{nm}$ with some bridging issues that can be attributed resist with the application of the under-layer. High sensitivity at hp $45 \mathrm{~nm}$ was maintained at 7.1 $\mathrm{mJ} / \mathrm{cm}^{2}$, with almost the same LWR at $10 \mathrm{~nm}$. However, an obvious improvement in pattern stability was observed at the $\mathrm{hp} 40 \mathrm{~nm}$ resolution limit with stable modulation of pattern sizes as low as hp $30 \mathrm{~nm}$.

3.3.2 Improvement the resist fomuration and prosses

We had the first improvement the resist materials that quencher was changed from THA to TOA. This resulted to improved resolution at hp 40nm with slight decrease of sensitivity and LWR. Next step, the protection group was replaced from isopinocampheroxymethyl to norbornanemethoxymethyl. Drastic improvement in sensitivity at $\mathrm{hp} 45 \mathrm{~nm}$ of $5.1 \mathrm{~mJ} \mathrm{~mJ} / \mathrm{cm}^{2}$ and partial resolution at hp 40nm was obtained. As a final step, we measured the dependece of PEB temperature that improved sensitivity and resolution at $\mathrm{hp}$ 40nm with slight decrease of LWR (Figure 4). 


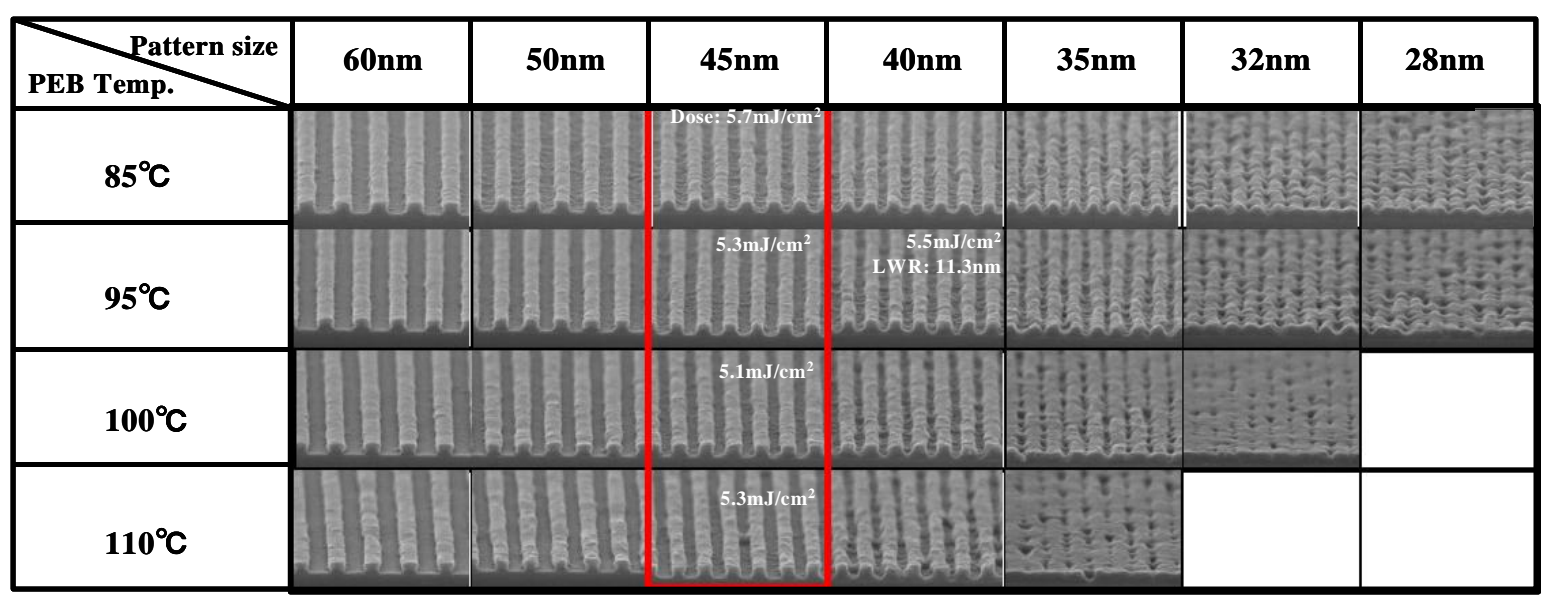

Fig. 4. The lithographic performance of the TFE/NB1FVIP based EUV resist.

\section{Conclusion}

Initial investigations on the application of TFE/NB1FVIP based resists for EUV lithography was performed were reported in 2009. High sensitivity and satisfactory resolution limit was achieved. However, these initial TFE/NB1FVIP based resist results show to need the improvement of high sensitivity, high resolution and low LWR for use EUV resists. It is clear that further material and process optimizations may be necessary to improve the present lithographic results.

\section{Acknowledments}

A part of this work is supported by New Energy and Industrial Technology Development Organization (NEDO). The authors would like to thank the Selete member companies (EUVLithomask Program) for the continued support. Takuji Ishikawa and Yuji Kume at the chemical division in Daikin Ind. Ltd. for synthesizing the polymers and monomers and polymer characterization.

\section{References}

1. K. Tawarayama, H. Aoyama, S. Magoshi, Y. Tanaka, S. Shirai, and H. Tanaka: Proc. SPIE 7271 (2009) 727118.

2. O. Wood, C.-S. Koay, K. Petrillo, H. Mizuno, S. Raghunathan, J. Arnold, D. Horak, M. Burkhardt, G. McIntyre, Y. Deng, B. La Fontaine, U. Okoroanyanwu, A. Tchikoulaeva, T. Wallow, J. H.-C. Chen, M. Colburn,S. S.-C. Fan, B. Haran, and Y. Yin: Proc. SPIE 7271 (2009) 727104

3. G. Vandentop, M. Chandhok, E. S. Putna, T. R. Younkin, J. S. Clarke, S.Carson, A. Myers, M. Leeson, G. Zhang, T. Liang, and T. Murachi: Proc.SPIE 7271 (2009) 727116.

4. J.-O. Park, C. Koh, D. Goo, I. Kim, C. Park, J.
Lee, J. Park, J. Yeo, S.-W.Choi, and C.-H. Park: Proc. SPIE 7271 (2009) 727114.

5. H. Meiling, N. Buzing, K. Cummings, N. Harned, B. Hultermans, R. de Jonge, B. Kessels, P. Ku“ rz, S. Lok, M. Lowisch, J. Mallman, B. Pierson, C. Wagner, A. van Dijk, E. van Setten, and J. Zimmerman: Proc. SPIE 7271 (2009) 727102.

6. M. Yoshioka, D. Bolshukhin, M. Corthout, G. H. Derra, S. Go"tze, J. Jonkers, J. Kleinschmidt, R. Mu“ ller, M. C. Schürmann, G. Schriever, R. Snijkers, and P. Zink: Proc. SPIE 7271 (2009) 727109.

7. A. Endo, H. Komori, Y. Ueno, K. M. Nowak, Y. Takayuki, Y. Tatsuya, T. Suganuma, T. Asayama, H. Someya, H. Hoshino, M. Nakano, M. Moriya,T. Nishisaka, T. Abe, A. Sumitani, H. Nagano, Y. Sasaki, S. Nagai, Y.Watanabe, G. Soumagne, T. Ishihara, O. Wakabayashi, K. Kakizaki, and H. Mizoguchi: Proc. SPIE 7271 (2009) 727108.

8. T. Miura, K. Murakami, H. Kawai, Y. Kohama, K. Morita, K. Hada, and Y. Ohkubo: Proc. SPIE 7271 (2009) 72711X.

9. J. V. Hermans, B. Baudemprez, G. Lorusso, E. Hendrickx, A. van Dijk, R. Jonckheere, and A.-M. Goethals: Proc. SPIE 7271 (2009) 72710T.

10. T. Terasawa, T. Yamane, T. Tanaka, T. Iwasaki, O. Suga, and T. Tomie:Jpn. J. Appl. Phys. 48 (2009) 06FA04.

11. C. Koh, L. Ren, J. Georger, F. Goodwin, S. Wurm, B. Pierson, J.-O. Park, T. Wallow, T. R. Younkin, and P. Naulleau: Proc. SPIE 7271 (2009) 727124.

12. H. Oizumi, D. Kawamura, K. Kaneyama, S. Kobayashi, and T. Itani:Proc. SPIE 7273 (2009) 72731M.

13. E. S. Putna, T. R. Younkin, M. Chandhok, and K. Frasure: Proc. SPIE 7273 (2009) 72731L.

14. International Technology Roadmap for Semiconductors 2007 Edition,Lithography.

15. T. Kozawa and S. Tagawa: Jpn. J. Appl. Phys. 
47 (2008) 8328.

16. H. Oizumi, T. Kumise, and T. Itani: J. Vac. Sci. Technol. B 26 (2008) 2252.

17. H. Oizumi, T. Kumise, and T. Itani: $J$. Photopolym. Sci. Technol. 21 (2008) 443.

18. M. Wang, C. T. Lee, C. Henderson, W. Yueh, J. M. Roberts, and K Gonsalves: Proc. SPIE $\mathbf{6 9 2 3}$ (2008) 692312.

19. T. Watanabe and H. Kinoshita: J. Photopolym. Sci. Technol. 20 (2007) 373.

20. H. Yamamoto, T. Kozawa, S. Tagawa, H. Yukawa, M. Sato, and J. Onodera: Appl. Phys. Express 1 (2008) 047001.

21. H. Ito and C. G. Willson: Polym. Eng. Sci. 23 (1983) 1012.

22. S. Nagahara, Y. Sakurai, M. Wakita, Y. Yamamoto, S. Tagawa, M. Komuro, E. Yano, and S. Okazaki: Proc. SPIE 3999 (2000) 386.

23. T. Kozawa, S. Tagawa, J. J. Santillan, M. Toriumi, and T. Itani: Jpn. J. Appl. Phys. 47 (2008) 4465.

24. R. Gronheid, C. Fonseca, M. J. Leeson, J. R. Adams, J. R. Strahan, C. G. Willson, and B. W. Smith: Proc. SPIE 7273 (2009) 727332.

25. T. Hagiwara, T. Furukawa, T. Itani, K. Fujii, T. Ishikawa, M. Koh, T. Kodani, T. Moriya, T. Yamashita, T. Araki, M. Toriumi, and H. Aoyama: Proc. SPIE 5376 (2004) 159.

26. F. Houlihan, R. Sakamuri, A. Romano, D. Rentkiewicz, R. Dammel, W. Conley, D. Miller, M. Sebald, N. Stepanenko, M. Markert, U. Mierau, I. Vermeir, C. Hohle, T. Itani, M. Shigematsu, and E. Kawaguchi: Proc. SPIE 5376 (2004) 134.

27. T. Ishikawa, T. Kodani, M. Koh, T. Moriya, T. Araki, H. Aoyama, T.Yamashita, M. Toriumi, T. Hagiwara, T. Furukawa, T. Itani, and K. Fujii:Proc. SPIE 5376 (2004) 169.

28. T. Itani, M. Toriumi, T. Naito, S. Ishikawa, S. Miyoshi, T. Yamazaki, and M. Watanabe: J. Vac. Sci. Technol. B 19 (2001) 2705.

29. J. J. Santillan, S. Kobayashi, and T. Itani: Jpn. J. Appl. Phys. 47 (2008) 4922.

30. N. Matsuzawa, H. Oizumi, S. Mori, S. Irie, E. Yano, S. Okazaki, and A. Ishitani: J. Photopolym. Sci. Technol. 12 (1999) 571.

31. S. Irie, T. Hagiwara, K. Fujii, Y. Itakura, Y. Kawasa, K. Egawa, I.Uchino, A. Sumitani, and T. Itani: J. Vac. Sci. Technol. B 22 (2004) 3513.

32. H. Xu, J. M. Blackwell, T. R. Younkin, and K. Min: Proc. SPIE 7273(2009) 72731, J.Jpn. J. Appl. Phys. 49 (2010) 06GF01 J. J. Santillan et al.06GF01-4 \# 2010 The Japan Society of Applied Physics
33.T. Hagiwara, T. Furukawa, T. Itani, K. Fujii, T. Ishikawa, M. Koh, T. Kodani, T. Moriya, T. Yamashita, T. Araki, M. Toriumi, H. Aoyama, "Characterization of TFE/norbornene-based fluoropolymer resist for 157-nm lithography," Proc. SPIE 5376, 159 (2004). M. Koh, T. Ishikawa, T. Araki, H. Aoyama, T. Yamashita, T. Yamazaki, H. Watanabe, M. Toriumi, and T. Itani, "The study on dry etch resistance of Fluorine functionalized polymers," Proc. SPIE 4690, 486 (2002). M. Koh, T. Ishikawa, T. Araki, H. Aoyama, T. Yamashita, T. Yamazaki, H. Watanabe, M. Toriumi, and T. Itani, "Synthesis of novel fluorinated norbornene derivatives for $157 \mathrm{~nm}$ application," Proc. SPIE 5039, 589 (2003). M. Toriumi, M. Koh, T. Ishikawa, T. Araki, H. Aoyama, T. Yamashita, T. Yamazaki, H. Watanabe, and T. Itani, "Novel Main-chain-Fluorinated Polymers for 157-nm Photoresists," Proc. SPIE 5039, 53 (2003). T. Ishikawa, T. Kodani, M. Koh, T. Moriya, T. Araki, H. Aoyama, T. Yamashita, M. Toriumi, T. Hagiwara, T. Furukawa, T. Itani, K. Fujii, " The dissolution behavior of tetrafluoroethylene-based fluoropolymers for 157-nm resist materials," Proc. SPIE 5376, 169 (2004).

34. M. Toriumi, T. Ohfuji, M. Endo, and H. Morimoto, "Swelling Analysis of Methacrylate Polymers in Aqueous Alkaline Developer," $J$. Photopolymer Sci. Technol. 12, 545 (1999); M. Toriumi, S. Ishikawa, and T. Itani, "Resist polymer dissolution study using Quartz Crystal Microbalance method," in Forefront of Lithographic Materials Research, Society of Plastic Engineers, Mid Hudson Section, pp. 271-281 (2001); M. Toriumi, T. Itani, J. Yamashita, T. Sekine, and K. Nakatani, "Dissolution characteristics of resist polymers studied by the Quartz Crystal Microbalance transmission-line analysis and the pKa acidity analysis", Proc. SPIE 4690904 (2002) and references therein.

35. T. Yamashita, T. Ishikawa, T. Yoshida, $T$. Hayami, T. Araki, H. Aoyama, T. Hagiwara, T. Itani, K. Fujii, " Synthesis of fluorinated materials for 193-nm immersion lithography and 157-nm lithography," Proc. SPIE 5753, 564 (2005). T. Yamashita, T. Ishikawa, T. Yoshida, T. Hayami, T. Araki, H. Aoyama, T. Hagiwara, T. Itani, K. Fujii," Synthesis of fluorinated materials for 193-nm immersion lithography and 157-nm lithography," J. Photopolymer Sci. Technol. 18, 631 (2005). T. Yamashita, T. Ishikawa, T. Yoshida, T. Hayami, H. Aoyama, "Novel fluorinated polymers for application in 193-nm lithography 
and 193-nm immersion lithography "Proc. SPIE 6153-81 (2006). K. Ishiwari, A. Ohmori, S. Koizumi, Nippon Kagaku Kaishi, 10,1942 (1985). Nippon Koukai Tokkyo Kouhou, JP2003-40840, JP2005-206587, JP2005-239710, JP2007-204385

D. Sanders, L. Sundberg, R. Sooriyakumaran, P. Brock, R. DiPietro, H. Truong, D. Miller, M.
Lawson, R. Allen, "Fluoroalchohol Materials with Tailored Interfacial Propaties for Immersion Lithography," Proc. SPIE 6519, 651904-1 (2007). M. Morita, H. Ogisu, M. Kubo, "Surface properties of perfluoroalkylethyl acrylate/n-alkyl acrylate copolymers", J. Applied Polymer Science, 73 (19), 1741 (1999). 